Dario Vinci

Ufficio Tutele Metropolitano presso il Comune di Bologna (Metropolitan Protection Office in the Municipality of Bologna, Italy) e-mail: dario.vinci@comune.bologna.it

ORCID: 0000-0002-0082-1111

DOI: $10.15290 /$ mhi.2020.19.01.15

\title{
Il MSNA, tra diritti consolidati e nascenti esigenze di tutela. Il punto di vista di un Ufficio Tutele Metropolitano
}

\begin{abstract}
Unaccompanied Foreign Minor, between consolidated rights and emerging protection needs. The point of view of an Italian Metropolitan Protection Office

The Italian Juvenile Law guarantees protection to unaccompanied foreign minors, which is a child coming from non-EU countries without parents. This phenomenon, in recent years, concerns the migratory flows of refugees, who arrive from Turkey or Libya to the coasts of Italy; the journey is often dramatic and sometimes it requires to pass through other European countries. The EU Regulation - Dublin III, which entered into force on 1 January 2014, points at the competence of the EU State in which the minors arrived at first; further transfers, except the need to be reunited with family members in other EU States, are not significant for the European law. The current system of granting refugee or other forms of international protection is based on this fundamental principle. The protection of unaccompanied foreign minors in Italy is achieved through a form of "multidisciplinary" support; the legal data is only one component. In addition, there are social and educational projects as well as protection for health; the need of an effective integration is perceived. Together with the social intervention, a legal public representative, the guardian, is appointed for unaccompanied minor. The competence belongs to the Municipality in which the minor is found or where, following a first reception, will be transferred on the basis of a regional territorial assignment, within the national system of Italian reception for refugee minors, called "SIPROIMI". The legal guardian's role is to
\end{abstract}


guarantee children's rights and an adequate assistance. It's a very thorny topic in the Metropolitan Area of Bologna, which has more than a million inhabitants; all the local offices created a "Public Metropolitan Protection Office" with the aims to manage these aspects. Promoting the function of these public offices confers the role of a "third party" in the protection of the children with the assistance of social and health services.

Key words: minor, refugee, guardian, Public Metropolitan Protection Office

\section{Introduzione $e^{1}$}

Ai sensi dell'art. 343 c. 1 c.c. "se entrambi i genitori sono morti o per altre cause non possono esercitare la responsabilità genitoriale, si apre la tutela".

Ai sensi poi dell'art. 354 c.c. "La tutela dei minori, che non hanno nel luogo del loro domicilio parenti conosciuti o capaci di esercitare l'ufficio di tutore, può essere deferita dal giudice tutelare a un ente di assistenza nel comune dove ha domicilio il minore o all'ospizio in cui questi è ricoverato. L'amministrazione dell'ente o dell'ospizio delega uno dei propri membri a esercitare le funzioni di tutela".

Tra le diverse cause per cui l'Autorità Giudiziaria è tenuta a nominare un Tutore abbiamo quella del minorenne straniero non accompagnato presente sul territorio italiaNo. La peculiarità dei destinatari di questa forma di tutela comporta che il Giudice Tutelare (in questo caso il Tribunale per i Minorenni, ai sensi del D.lgs 220/17) non possa nominare quasi mani familiari del minorenne quali figure tutelari, dovendo invece nominare un Ente Pubblico oppure, come solo di recente previsto, un Tutore privato volontario.

Quando la Tutela è deferita ad un Ente Pubblico, di solito il Comune presso cui il minorenne è preso in carico e spesso insiste anche il luogo (es. Comunità o famiglia affidataria) che lo accoglie, la Tutela si dice Pubblica.

Al Tutore competono attività di promozione e garanzia dei diritti del soggetto tutelato, quindi non soltanto di tipo conservativo (preservare diritti già

1 L'Ufficio Tutele Metropolitano è presente presso il Comune di Bologna (Area Welfare e Promozione del Benessere della Comunità), che attivato in via sperimentale nel 2017 a Bologna, quale esempio tra i pochi in Italia, cura per l'intera Area della Città Metropolitana gli aspetti giuridici in materia di Pubbliche Tutele, tra cui quelle aperte a favore dei minorenni stranieri non accompagnati, promuovendo in raccordo con l'Azienda comunale cittadina (ASP Città di Bologna) che ha in carico sociale i MSNA, l'Ufficio del Garante Regionale per l'Infanzia e l'Adolescenza ed il Tribunale per i Minorenni anche il recente Istituto della Tutela Volontaria. Copre l'ambito territoriale dei Servizi Sociali afferenti i Distretti AUSL di Bologna città, San Lazzaro, Casalecchio Lavino e Valsamoggia, Appennino, Pianura Est, Pianura Ovest, Imola. 
acquisiti) ma anche di stimolo ed impulso perché il tutelato (nel linguaggio civilistico classico lo chiameremmo "pupillo") riceva diritti prima non goduti, avendone però titolarità (es. assegni sociali, residenza, iscrizione al servizio sanitario nazionale, ecc).

Vi sono poi i Garanti per l'Infanzia e l'Adolescenza, che a livello nazionale e regionale promuovono i diritti minorili a livello più politico, sollecitando ad esempio tutele giuridiche non ancora vigenti, ma ritenute utili da riconoscere ai minorenni.

Al contempo, l'Ente Pubblico, per altre fonti normative tra cui la Legge quadro sull'assistenza sociale (n. 328/00), è anche titolare degli interventi sociali a favore di questi soggetti bisognosi non solo di una tutela giuridica ma anche sociale (oltrechè sanitaria, educativa, ecc.).

In proposito ai sensi dell'art.315 bis cc: Il figlio ha diritto di essere mantenuto, educato, istruito e assistito moralmente dai genitori, nel rispetto delle sue capacità, delle sue inclinazioni naturali e delle sue aspirazioni.

Mentre per l'art. 30 Cost. c. 2 "nei casi di incapacità dei genitori, la legge provvede a che siano assolti i loro compiti".

Di qui la necessità che il minorenne straniero non accompagnato debba essere assistito, con una competenza mista Stato (Ministero degli Interni) /Regioni ed Enti Locali attraverso le forme tipiche del diritto minorile, in primis la Legge 184/83 in materia di affidamento familiare, per cui ex art. 2 "Il minore temporaneamente privo di un ambiente familiare idoneo, nonostante gli interventi di sostegno e aiuto disposti ai sensi dell'art. 1, è affidato ad una famiglia... ove non sia possibile è consentito l'inserimento del minore in una comunità di tipo familiare...".

Un sistema di accoglienza a competenza sociale in materia di immigrazione affidata allo Stato, e gestita o direttamente dal Ministero dell'Interno a mezzo di una "Struttura di Missione" con raccordi con i singoli Enti locali e le strutture di accoglienza dislocate sul territorio aderenti alla rete ex Sprar (Sistema di Protezione per Richiedenti Asilo e Rifugiati), ora chiamata SIPROIMI (Sistema di protezione per titolari di protezione internazionale e per minori stranieri non accompagnati), o direttamente dai Comuni che aderiscono a progetti Europei FAMI ("Fondo asilo migrazione e integrazione 2014-2020 Fami)", ovvero uno strumento finanziario istituito con Regolamento UE n. 516/2014 con l'obiettivo di promuovere una gestione integrata dei flussi migratori sostenendo tutti gli aspetti del fenomeno: asilo, integrazione e rimpatrio.

Quindi il ruolo del Tutore, in particolare quando Pubblico, è in stretto raccordo con chi governa gli interventi a sostegno del minore, tra cui la sua accoglienza. Il che non può però portare ad una sovrapposizione tra ruoli ontologicamente distinti, ed anzi retti non solo da collaborazioni ma anche da dinamiche di garanzia e di controllo reciproci. 
Tale assetto funzionale, nella dicotomia tutela giuridica/tutela sociale, nel tempo si è reso ancora più complesso perché in tema di presa in carico sociale il Legislatore ha introdotto gli Istituti dell'affidamento familiare e dell'adozione (1. 184/83), oltrechè una nuova concezione di Welfare (L. 328/00) e di un rafforzamento dell'Autorità Giudiziaria minorile, con sempre maggiori poteri riconosciuti in materia di protezione dell'infanzia (dalla riforma della L.151/75 a quelle ex L.219/12 e D.lgs 154/13); tutte novità che hanno contaminato qualitativamente e quantitativamente gli interventi tutelari conseguenti (si pensi alle tutele aperte a favore di minorenni interessati da procedure volte ad adottarli, oppure a seguito di provvedimenti ablativi delle responsabilità genitoriali o ancora ai fenomeni migratori recenti che introducono la tutela non solo quale conseguenza di nuovi fenomeni geopolitici ma anche per una nuova cultura del diritto minorile).

Le recenti modifiche normative in materia di filiazione (1. 219/12, D.lgs 154/13) e l'utilizzo sempre più diffuso dei moderni modelli a protezione giuridica delle persone prive in tutto o in parte di autonomia, quale i minorenni, tra cui quelli stranieri non accompagnati, in ambito tutelare o gli anziani in materia di Amministrazione di Sostegno (1. 06/04), introducono inoltre da un lato nuovi diritti in materia di minori, famiglia e tutela dei soggetti deboli, anche maggiorenni, e dall'altro nuove complessità giuridiche per gli Enti Locali che direttamente o a mezzo di deleghe all'AUSL, Unioni di Comuni ovvero ASP risultino titolari di tali prese in carico, da cui discendono doveri di legge e conseguenti responsabilità, tanto dell'Amministrazione quanto dei singoli professionisti coinvolti.

Lo stesso Legislatore, presta infatti sempre più attenzione ai temi della responsabilità civile derivante dalla custodia di minori e adulti incapaci (artt. 2043, 2047 e 2048 cc), degli obblighi di riservatezza e del corretto trattamento dei dati personali, sensibili, sanitari e giudiziari ma anche degli obblighi connessi in capo agli operatori socio-sanitari tra cui quelli in ambito civile e penale.

Anche a livello processuale, si assiste a nuove geografie giudiziarie, che interessano tanto il Giudice Tutelare, che il Tribunale Ordinario in sede collegiale o anche monocratica quanto il Tribunale per i Minorenni. Ciò sia in materia di minorenni già presenti in Italia che a quelli giunti dall'estero, in particolari da spazi extra Ue, i cd Minori Stranieri non Accompagnati; questi ultimi spesso titolari anche di protezioni internazionali e quindi bisognosi di tutele giuridiche specifiche.

Chiudono lo scenario, le nascenti ed anzi ormai consolidate figure di Garanzia, a livello nazionale e territoriale, tra cui il Difensore civico, comunale o regionale ed il Garante per l'Infanzia e l'Adolescenza, nazionale o regionale, che impongono nuovi modelli di raccordo. 
Sullo sfondo, le riforme dettate in materia di processo civile telematico e di digitalizzazione della Pubblica Amministrazione, che richiedono processi condivisi a livello metropolitano, poiché afferenti alle stesse Autorità Giudiziarie e ai medesimi Organi di garanzia.

La necessità degli Enti titolari delle funzioni socio-sanitarie di raccordarsi il più possibilmente a livello unitario, ad esempio a livello di Città Metropolitana, nel creare nuove prassi amministrative e procedurali e di raccordo con le AAGG competenti e gli organi di Garanzia, è pertanto attuale. In particolare nel caso in cui gli utenti in carico siano interessati anche da una Pubblica Tutela o Curatela o comunque si trovino in situazioni di particolare disagio per cui potrebbero risultare futuri beneficiari, siano essi minori adulti o anziani, di quelle che vengono chiamate dal Legislatore "forme di protezione giuridica" e quindi, tutela, curatela o amministrazione di sostegno.

In questo caso, alle necessità sopra evidenziate, si aggiunge l'utilità per gli Enti titolari di Tutela, Curatela o dei doveri in tema di Amministrazione di Sostegno, di avere un modello organizzativo che preveda un adeguato supporto giuridico all'Ufficio del Pubblico Tutore e agli operatori sociali coinvolti, atteso che tali nomine sono spesso indice di particolare complessità (procedimento di adottabilità, reati con vittime minorenni, conflitti o fragilità familiari importanti con conseguente complessità sociale e giuridica).

Ciò tanto con riguardo ai minorenni, cui è demandata una tutela in capo all'Ente sociale di residenza o domicilio, in forza di provvedimenti ablativi della responsabilità genitoriale ovvero nell'assenza o impedimento dei soggetti che in via ordinaria sarebbero titolari della stessa (es i minori stranieri non accompagnati) quanto per soggetti maggiorenni, poiché limitati giudizialmente delle loro capacità di agire $\mathrm{o}$ ancora nei casi di interdizione legale per una condanna penale.

Attenzione andrà poi rivolta ai recenti modelli sperimentali volti a trasformare, in alcuni ambiti, le Pubbliche Tutele in Tutele Volontarie (1. 47/17 in materia di minori stranieri non accompagnati), nonché alle forme di volontariato a sviluppo dell'Istituto dell'Amministrazione di Sostegno anche per i casi ad oggi demandati in via residuale alle Pubbliche Amministrazioni.

Con ciò, l'attività dei Servizi Pubblici dovrà offrire risposte ma da una prospettiva diversa, all'interno del concetto di Welfare di Comunità, garantendo una stretta integrazione tra servizi sociali, gestori del servizio e volontari.

Per il D.lgs. 220/2017 la competenza a nominare un Tutore ai MSNA e a gestirne tutte le attività tutelari, tra cui l'accertamento dell'età, i provvedimenti relativi all'eventuale rimpatrio o coesione familiare in Paesi terzi, la rettifica delle generalità, il prosieguo amministrativo, ecc. spetta non più al Giudice Tutelare presso il Tribunale Ordinario, bensì al Tribunale per i Minorenni. 
Nel frattempo la Corte di Cassazione, con ordinanza 5 marzo-3 aprile 2019 n. 9199 ha avuto modo di chiarire che per MSNA devono intendersi anche i minorenni che hanno parenti in Italia diversi dai genitori o del tutore (i soli quindi che potrebbero esercitare poteri di rappresentanza legale).

Il tema della tutela dei MSNA è affiancato dal nascente interesse per la figura dei cd Tutori volontari (disciplinati dalla citata L. 47/17 e già introdotti in via sperimentale anche negli anni precedenti , es Emilia Romagna con le esperienze "pilota" di Bologna, Ferrara e Reggio Emilia).

\section{L'esigenza di terzietà del (Pubblico) Tutore}

Il modello della Tutela Pubblica è entrato in crisi nel momento in cui questa (ed anche la Curatela), per le modifiche del sistema di Welfare intercorse dal codice civile del 1942 ad oggi, è stata estesa a situazioni nuove (es. ai minorenni stranieri non accompagnati o i cui genitori ricevono provvedimenti limitativi, sospensivi o ablativi della responsabilità genitoriale; $\mathrm{o}$ ancora a moderne forme di sostegno ai disabili, pazienti psichiatrici ed anziani) i cui titolari sono al contempo divenuti anche destinatari di precisi interventi sociali e sanitari.

Nel momento in cui lo stesso Ente funge tanto da Tutore che da erogatore di quei servizi alla persona al proprio tutelato, si è pertanto fatta strada la necessità di separare tali ruoli.

Nonostante infatti sia lo stesso codice civile all'art. 354 (approvato nel 1942 ed in verità di cui il primo codice qui interessato venne votato nel 1938), a prevedere espressamente la possibilità di nominare ad un soggetto bisognoso di protezione giuridica un tutore pubblico, va considerato che quando venne immaginata quella possibilità il diritto all'assistenza sociale mancava di normative importanti, quali il D.P.R. 617/77 (sul trasferimento delle competenza sociali ai Comuni), la L. 833/78 (sul SSN e con la possibilità di delegare alle ASL le competenze sociali);ma anche la 1. 184/83 sull'affidamento familiare e le adozioni), la 1. 328/00 (riforma dei servizi sociali), la 1. 6/04 (sull'Amministrazione di sostegno) ed ovviamente la Costituzione nel 1948 (poi riformata nel 2001 al titolo V parte II).

Attraverso l'articolato normativo sopra richiamato, possiamo pertanto affermare che per quanto oggi sia rimasta la possibilità che un Ente titolare della competenza socio-sanitaria venga nominato Tutore, nella pratica ciò rileva un rischio di incompatibilità o quantomeno di inopportunità, per il conflitto di interessi, astratto o concreto, a tale esercizio sotteso, arrivando a volte a fare coesistere sullo stesso soggetto Pubblico (e persino sul medesimo funzionario/ dirigente incaricato) posizioni distinte e non sempre tra di loro compatibili o conciliabili, perchè allo stesso tempo di "controllato" e "controllore", ovvero di "fruitore" ed "erogatore" della prestazione socio-sanitaria. 
Sia per gli utenti minorenni che maggiorenni, tra cui disabili e anziani, sono infatti diverse le occasioni nelle quali il Tutore pubblico rischia di assumere decisioni in conflitto di interessi, tanto su questioni civili (es. partecipazione a processo per accertamento di abbandono del minore, dove i servizi sociali e sanitari rivestono invece una distinta e diversa funzione processuale) quanto su tematiche sociali e patrimoniali (immaginiamo la neutralità di un tutore, nel caso di un intervento che comporti un collocamento in struttura, dove sarà tenuto a perseguire due diverse esigenze, non sempre tra loro conciliabili, quali il benessere del tutelato ma anche il contemperamento della spesa pubblica). Proprio la recente normativa sull'amministrazione di sostegno ha posto, quale principio generale (all'art. 408 c.c.), l'incompatibilità tra l'amministratore di sostegno nominato (forma di protezione giuridica al pari della tutela e della curatela) e gli Enti che ne curano la presa in carico sociale e sanitaria.

Di recente la Dottrina ha avuto modo di chiarire tale questione. Per Gionca$\mathrm{da}^{2}$ "in virtù del combinato disposto, la tutela può essere affidata formalmente ad Enti Pubblici nella persona del loro legale rappresentante salva l'incompatibilità concernente gli operatori che hanno direttamente in carico il disabile".

Sul punto Lavedini ${ }^{3}$ rileva che per quanto riguarda gli operatori (dipendenti dell'ente locale o di altri enti istituzionalmente competenti all'assistenza) direttamente coinvolti nell'assistenza del soggetto interessato, la norma appare alquanto chiara ed univoca nel senso della loro incompatibilità ad assumere la funzione di Amministratore di sostegno a favore dello stesso soggetto. Nel caso di soggetti beneficiari non direttamente assistiti dall'operatore, ma comunque posti alle cure o a carico dell'ente, l'incompatibilità si fonda sulla potenziale conflittualità degli interessi coinvolti, come sarà meglio chiarito nel prosieguo di questa trattazione.

Con riferimento ai dirigenti degli enti impegnati nell'assistenza, si può ritenere che la loro incompatibilità tragga una prima origine proprio dal divieto posto in capo agli operatori. Gli operatori dipendono direttamente dai loro dirigenti e ne eseguono le determinazioni. Ne deriva che se il Legislatore ha vietato la funzione di Amministratore di sostegno in capo agli operatori, non si può pensare che non sussista analoga incompatibilità in capo ai dirigenti i quali sono gli unici soggetti dell'ente ad avere un potere gestionale autonomo ed il potere di porre in essere atti di rilevanza esterna vincolanti per l'Ente".

Quanto introdotto dall'art. 424 c. 3 c.c., elevandolo a principio generale in materia di disabili, dovrà necessariamente riguardare anche l'ambito minorile,

2 M. Gioncada, Diritto dei servizi sociali. Per assistenti sociali, psicologi, educatori, avvocati, Maggioli 2012, p. 368

3 G. Lavedini, Prime brevi considerazioni sul ruolo dell'Ente Locale nell'Amministrazione di Sostegno, "Il Diritto della Regione" 2005, n. 1-2. 
dove tale distinzione dei ruoli tra tutore e servizio sociale è chiaro a partire dalla legge 184/83 (modificata dalla L. 149/01) in materia di affidamento e adozione, dove sono appunto distinte le funzioni di queste due figure (art. 4, 12 e 15), non potendole sovrapporre, in particolare allorquando il Tutore diviene figura di garanzia e pertanto anche di controllo rispetto all'operato degli stessi servizi sociali, stante una autonoma legittimità processuale oltrechè di rappresentanza legale del minorenne tutelato.

Lo stesso articolo 354 c.c. per Pazè deve essere interpretato in tal modo poichè "questa forma di tutela, deferita ad un ente è ancora in vita, ovviamente con adattamenti, nel senso che vengono nominati alla tutela il Comune o la USL nel cui territorio vivono il minore o l'incapace, rispettivamente nella persona del sindaco o del presidente, con possibilità di delega" ed ancora " gli enti locali e i servizi che da essi dipendono, sono tenuti ad esercitare una funzione di controllo politico e gestione dell'assistenza, incompatibile con il fatto di essere essi stessi tutori"4.

Nel solco di tale ragionamento, Strumendo, peraltro nell'ambito di una pubblicazione del difensore civico regionale ER del 2011, "per quanto riguarda la possibile incompatibilità o difficoltà nel rapporto fra le competenze, le funzioni e le responsabilità degli operatori professionali dei servizi e l'attività di affidamento o tutela, ritiene che il potenziale pericolo in effetti esista, ma al tempo stesso debba essere assolutamente vietato" ${ }^{\text {. }}$.

Si è pertanto diffusa in questi anni, sia in ambito sociale che sanitario, l'esigenza di una terzietà della funzione del Tutore rispetto al servizio sociale o socio sanitario, che hanno la presa in carico del soggetto, minorenne o interdetto, giudiziale o legale, privilegiando elementi di garanzia della funzione tutoria (e curatoria).

Come detto, laddove il Tutore (o anche Curatore) Pubblico risulti coincidente con chi ha la responsabilità della erogazione dei servizi sociali a favore dei minori, disabili, pazienti psichiatrici, anziani e carcerati si pone un possibile conflitto di interesse, quantomeno in astratto, o comunque una difficile e potenzialmente ambigua gestione dei due ruoli insieme. Ciò in particolare se in capo alla stessa figura apicale o comunque allo stesso Settore o Dipartimento.

Alcuni esempi:

In ambito minorile:

conflitto di interessi potenziale tra la rappresentanza legale nell'ambito di un processo civile in tema di responsabilità genitoriale, come anche nella gestione della progettualità sociale.

4 P. Pazè, Tutela e Curatela dei minori, in Trattato di Diritto di Famiglia, dir. P. Zatti, Milano 2002.

5 L. Strumendo, Intervento in materia tutelare, "Pubblicazione Difensore Civico Emilia Romagna Quaderno" 2011, N. $2 / 2011$. 
Il tutore, in quanto legale rappresentante deve garantire che nel procedimento giudiziale prevalga l'interesse del minore, assumendo pertanto una posizione fisiologicamente distinta da quella dei servizi sociali, coi quali potrebbe essere persino in disaccordo, ma soprattutto di censura verso le condotte tenute dai genitori dello stesso minorenne.

Diverso è invece il ruolo dei servizi sociali e sanitari, che occupandosi della presa in carico debbono invece poter stabilire una diversa dialettica con i genitori medesimi, conquistandone possibilmente la fiducia, nonostante l'eventuale inadeguatezza, per promuovere miglioramenti;

tanto che la 1. 184/83 distingue, in materia di accertamento dello stato di abbandono, anche a livello processuale il ruolo dei servizi socio-sanitari e del Tutore. Solo a quest'ultimo compete di nominare un avvocato al minorenne, nonché di presenziare obbligatoriamente in udienza. Non avendo all'opposto i Servizi il ruolo di parte processuale.

In materia di disabili adulti:

- anche nella materia degli adulti, nelle procedure interdittive e di nomina di Amministratore di Sostegno, valgono i distinguo processuali già introdotti per i minorenni, tra competenze dei Servizi alla persona e ruolo di legale rappresentante (o Ente promotore una ADS), e quindi con posizioni non necessariamente coincidenti con i primi, da parte del Tutore (o dell'Ente promotore dell'ADS).

Lo stesso dicasi in merito alla cura e al patrimonio dell'interdetto, le funzioni sono diverse. La promozione della prima e la gestione della seconda sono di competenza propria del Tutore, o dell'Amministratore di Sostegno, di cui l'Ente Pubblico rimane pur sempre promotore e quindi con una posizione di garanzia.

Funzione che andrebbe separata dunque dall'Ente titolare dell'erogazione dei Servizi socio-sanitari e della relativa riscossione di rette, per tutte le tipologie di interdetti, giudiziali e legali (disabili, pazienti psichiatrici, anziani, ma anche carcerati).

Tanto che la normativa in materia di Amministrazione di Sostegno indica espressamente, all'art. 408 c.c. che "non possono ricoprire le funzioni di amministratore di sostegno gli operatori dei servizi pubblici o privati che hanno in cura o in carico il beneficiario".

\section{Le Tutele dei MSNA}

Come detto, tra i casi recenti che richiedono la nomina di un Tutore troviamo i minorenni stranieri non accompagnati. Questi vengono dapprima indicati con tale terminologia ad opera del cd decreto Martelli (n. 416/89 poi convertito con legge n. 39/90) e dei successivi atti normativi ex artt. 32 e 33 del T.U. Imm 
(D.Lgs. n. 286/1998) e Reg. Att. (D.P.R. n. 394/1999). E' però nel D.P.C.M. n. 535 del 1999 che all'art. 1, co. 2, ne viene data la prima definizione "Per „minore straniero non accompagnato presente nel territorio dello Stato", di seguito denominato „minore presente non accompagnato“, s'intende il minorenne non avente cittadinanza italiana o di altri Stati dell'Unione europea che, non avendo presentato domanda di asilo, si trova per qualsiasi causa nel territorio dello Stato privo di assistenza e rappresentanza da parte dei genitori o di altri adulti per lui legalmente responsabili in base alle leggi vigenti nell'ordinamento italiano".

Questa formulazione viene poi sostituita solo nel 2017, con l'entrata in vigore della Legge 47 che all'art. 2 recita "per minore straniero non accompagnato presente nel territorio dello Stato si intende il minorenne non avente cittadinanza italiana o dell'Unione europea che si trova per qualsiasi causa nel territorio dello Stato o che e $e^{c}$ altrimenti sottoposto alla giurisdizione italiana, privo di assistenza e di rappresentanza da parte dei genitori o di altri adulti per lui legalmente responsabili in base alle leggi vigenti nell'ordinamento italiano".

Ai sensi di legge (D.P.R. 616/77, art. 320/00, D.lgs 112/98; art. 117 e 118 Cost.) la presa in carico sociale è di competenza dei Comuni, attraverso le forme organizzative individuate (gestione diretta, delega all'AUSL, Aziende Pubbliche di Servizi alla Persona (cd. ASP), Consorzi, Unioni di Comuni, ecc.).

La Materia dell'Immigrazione però è di competenza statale ai sensi dei richiamati articoli della Costituzione.

La scelta se del fenomeno dei minorenni stranieri non accompagnati dovesse prevalere la loro condizione di minorenne da tutelare (solo sul territorio italiano) ovvero di soggetto straniero (irregolarmente presente) è stata pacificamente risolta nel primo senso, anche alla luce della Convezione dei Diritti del Fanciullo, sottoscritta dall'Italia a New York il 20 novembre nel 1989 e ratificata con L. 176/91. Ciò è chiaro in particolare all'art. 19 del TU Immigrazione (D.lgs 286/98) per cui i minorenni stranieri non accompagnati non possono essere rimpatriati o comunque respinti, salvo casi eccezionali. Ed anche nel caso in cui fosse necessario (per ragioni di pubblica sicurezza o perché il minore è scappato da casa e vuole farvi rientro) è sempre essenziale un provvedimento autorizzatorio di un Giudice, ora il Tribunale per i Minorenni su impulso della locale Procura Minorile.

Questa natura ibrida dello straniero irregolare ma minorenne non accompagnato ha però subito nel corso del tempo degli altalenanti approcci politici in materia di definizione dei progetti destinati a tali soggetti. In proposito, valorizzare o meno i progetti di accoglienza a questi ragazzi e ragazze dedicati, come anche la possibilità più o meno agevole e strutturata di permettere loro una conversione del permesso di soggiorno alla maggiore età come l'accesso ai Servizi Pubblici, tra cui sanità, istruzione, formazione professionale, corsi di alfabetizzazione, partecipazione a gruppi socio-educativi, allo sport, ecc. diveniva 
cartina di tornasole tra due modelli opposti: un'accoglienza adempimentale/situazionale ed una effettiva/sistemica, propria del paradigma integrativo, inteso peraltro anche quale nuova forma di prevenzione in materia di sicurezza.

Solo un sistema di Welfare e di accoglienza che fa promuovere la permanenza del neo-maggiorenne all'interno della nazione che lo ha accolto da minorenne, può beneficare dei frutti sociali di tale accoglienza, trasformando la spesa in investimento. Ciò anche in termini di sicurezza sociale e prevenzione.

\section{Il Tutore volontario}

Ai sensi dell'art. 11 L. 47/17 "presso ogni tribunale per i minorenni e' istituito un elenco dei tutori volontari, a cui possono essere iscritti privati cittadini, selezionati e adeguatamente formati, da parte dei garanti regionali per l'infanzia e l'adolescenza, disponibili ad assumere la tutela di un minore straniero non accompagnato (fino ad un massimo di tre tutele ex D.lgs 220/17)".

Il Tutore Volontario, previsto dalla citata legge Zampa, è un cittadino che decide di fare da rappresentante legale del MSNA e di indirizzarlo nel percorso di crescita, educazione e integrazione.

Ai tutori volontari viene richiesto anche di stabilire un rapporto emotivo con i minori - che rimangono nelle strutture di accoglienza -, di ascolto, di aiuto nelle scelte, tale da restare vivo anche dopo il raggiungimento dei 18 anni.

Il tutore diventa in questo modo un punto di riferimento, un "genitore sociale", un portavoce per questi ragazzi.

Per le Linee guida del Garante Nazionale per l'Infanzia e l'Adolescenza, i requisiti per poter diventare tutori sono:

- Cittadinanza Italiana o di altro paese dell'Unione Europea, oppure di paese esterno all'Unione Europea purché si sia in possesso di permesso di soggiorno e si abbia conoscenza della lingua e cultura italiana, che verrà verificata in sede di selezione.

- Residenza in Italia.

- Età non inferiore ai 25 anni.

- Godimento dei diritti civili e politici.

- Assenza di condanne penali, di procedimenti penali o di procedimenti per l'applicazione di misure di sicurezza o prevenzione.

- Assenza delle condizioni di "incapacità all'ufficio tutelare" previste dalla legge (art. 350 Cod. Civ.).

- Ineccepibile condotta.

- Disponibilità di tempo ed energie per esercitare la funzione.

Il tutore viene nominato dal Tribunale per i Minorenni (mod. D.lgs 220/17) ed esercita, a titolo volontario e gratuito, la responsabilità genitoriale (disciplinata dal codice civile). 
Ha quindi la responsabilità di curare gli interessi e di perseguire il benessere del minore, che rappresenta negli atti e nei procedimenti con valore legale.

Il tutore volontario non convive con il minore né è tenuto al suo mantenimento, il suo ruolo peraltro benchè discenda dal diritto ha reale senso nella misura in cui si declina sul terreno sociale e di relazione con il tutelato. $\mathrm{Ma}$ non quale figura surrogatoria delle figure genitoriali che spesso esistono ma sono soltanto lontane dai figli ma quale "mentore", figura di riferimento e di supporto.

Le stesse esperienze dei Tutori volontari sono spesso frutto del lavoro congiunto tra Garanti Regioni, Servizi Sociali locali ed Uffici del Pubblico Tutore al fine di integrare la rete del Welfare territoriale con una nuova figura, assai peculiare perché esterna ai soggetti pubblici, una sorta di "microgarante".

Guardando all'ambito nazionale infatti vi sono alcune aree geografiche dove il Tutore volontario va a riempire un vuoto, una sofferenza dei Servizi Pubblici a garantire i ruoli tutelare e di presa in carico sociale; altre aree in cui invece il Sistema è strutturato, e capace di gestire in autonomia (differenziandole) le funzioni tutelari e sociali da riuscire a formare annualmente nuovi gruppi di tutori volontari.

Questi vengono seguiti per tutto il corso del ciclo formativo, incontrati singolarmente dal Servizio Sociale al punto di potere proporre (come nel caso dell'Emilia Romagna), un abbinamento specifico al Tribunale per i Minorenni e successivamente alla nomina incontrati periodicamente in gruppi di sostegno e confronto costante per rafforzarne le competenze e, se possibile, anche le motivazioni. In una logica di "manutenzione" delle relazioni sociali simile a quella dell'affidamento familiare.

Ciò comporta inoltre che chi è Tutore di un minorenne straniero non accompagnato a volte può chiedere di accogliere il "pupillo" alla maggiore età, all' interno di progettualità recenti, tra cui il progetto VESTA o anche semplicemente di forme di volontariato, tra cui la cd. "vicinanza solidale".

In questo Sistema, nel cui contesto i Servizi Sociali operano al fianco del Tutore volontario (così come avrebbero fatto con il Pubblico Tutore) le attività più complesse (regolarizzazione, iter del riconoscimento della protezione internazionale, incombenze burocratiche) possono essere delegate dal Tutore privato, in parte o in toto, all'Ufficio pubblico preposto. Non è escluso che lo stesso Ufficio comunale del Pubblico Tutore possa avere un ruolo di facilitatore in questo percorso, ad esempio partecipando a momenti di formazione giuridica. 


\section{L'Istituto della Tutela}

L' Istituto classico della TUTELA è stato adattato, anche a mezzo della riforma del 2017, al moderno fenomeno dei minorenni stranieri non accompagnati.

Dovremo però ripercorrere i principi codicistici per delineare i doveri principali in capo al Tutore.

Ai sensi dell'art. 357 c.c. "Il tutore ha la cura della persona del minore lo rappresenta in tutti gli atti civili e ne amministra i beni".

Ciò comporta una tripartizione: cura del minore rappresentanza negli atti civili, amministrazione dei beni.

1) Tra gli atti di cura troviamo le questioni di cd. "rilevanza interna della tutela, quindi convalidare il luogo di cura e di vita del minorenne (es. Comunità a famiglia affidataria), il mantenimento (di cui è però materialmente onerato il Servizio Sociale; si chiarisce anche che gli ordinari con scuola, sanità ed altre istituzioni sono di competenza del Servizio affidatario ai sensi dell' art. 5 della $1.184 / 83)$.

Possiamo pertanto dire che nel contesto di cura e vigilanza il Tutore, Pubblico o Privato esercita poteri di controllo piuttosto che di gestione diretta.

2) Tra gli atti di rappresentanza in tutti gli stati civili troviamo le questioni di cd "rilevanza esterna", ovvero che comportano una relazione del Tutore con soggetti terzi, nell' esercizio della rappresentanza legale sul minorenne. Tra cui i rapporti straordinari di cui sopra (es. consenso informato di tipo sanitario, tra cui i vaccini facoltativi e interruzione volontaria di gravidanza, TSO; iscrizione scolastica ad nuovo ciclo di studi; scelte religiose, interventi necessari del tutore in materia di matrimonio, affidamento familiare, accertamento stato di abbandono, adozione). Ed ancora, passaporto, carta identità, ISEE, privacy, istanza rifugio, residenza, sanità, percorsi formativi, godimenti dei diritti sociali di cui risulta titolare o possibile beneficiario.

In questa sfera di competenze abbiamo anche le rappresentanze processuali in sede penale, sia quando il minorenne è parte offesa (potendosi costituire in giudizio e persino quale parte civile, a mezzo di un legale nominato dal medesimo Tutore ex artt. 78 e $99 \mathrm{cpp}$ ) che quando questi si trovi a dovere subire un processo penale a mezzo delle regole del processo penale minorile, ex PROCESSO PENALE MINORILE D.P.R. 448/88 e D.LGS 272/89.

3) Tra gli atti di Amministrazione dei beni troviamo: gestione del patrimonio esistente, attività per ricevere entrate future, tra cui la richiesta degli assegni sociali (es indennità di frequenza, di accompagnamento, altri bonus sociali, borse di studio, ecc.). Alcune operazioni contabili (incasso o spese potrebbero essere soggette ad autorizzazione del Tribunale, quale Giudice Tutelare, ex artt. 372,374 e 375 c.c.). 


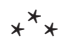

Le forme di Responsabilità giuridiche.

Le attività sopra richiamate sono doverose perché ne discendono responsabilità, di varia natura giuridica e correlate conseguenze normative.

- Responsabilità Civile: è una specifica responsabilità civile, prevista all' art. 2048 cc: "Il padre e la madre, o il tutore sono responsabili del danno cagionato dal fatto illecito dei figli minori non emancipati o delle persone soggette alla tutela, che abitano con essi. Le persone indicate dai commi precedenti sono liberate dalla responsabilità soltanto se provano di non aver potuto impedire il fatto".

Una responsabilità in cui l'onere della prova ricade sul Tutore ma che viene stemperata, nei fatti, dalla circostanza fisiologica che quasi mai il Tutore, pubblico o privato, ha in custodia il minorenne né partecipa al suo percorso educativo; motivo per cui queste figure tendono a beneficiare sistematicamente della scriminante dell'ultimo comma.

Tale principio vale anche in materia di sanzioni amministrative (tra cui le violazioni elevate dalla Polizia Locale o dalla Polizia per violazioni del codice della strada), come riporta l'art. 2 della L 689/81 "Non può essere assoggettato a sanzione amministrativa chi, al momento in cui ha commesso il fatto, non aveva compiuto i diciotto anni o non aveva, in base ai criteri indicati nel codice penale, la capacità di intendere e di volere, salvo che lo stato di incapacità non derivi da sua colpa o sia stato da lui preordinato".

Fuori dei casi previsti dall'ultima parte del precedente comma, della violazione risponde chi era tenuto alla sorveglianza dell'incapace, salvo che provi di non aver potuto impedire il fatto".

Si chiarisce che la responsabilità del Tutore per fatti illeciti commessi dal minorenne va distinta da quella ricondotta al medesimo tutore, in sede civile o penale, per condotte a danno del minorenne (di particolare rilievo quando riguardano un illecito penale, come di seguito.

- Responsabilità amministrativa: ai sensi dell'art. 382 c.c. "Il tutore deve amministrare il patrimonio del minore con la diligenza del buon padre di famiglia. Egli risponde verso il minore di ogni danno a lui cagionato violando i propri doveri”.

- Responsabilità penali: riguarda i reati commissivi ed omissivi (propri ed impropri) commessi dal Tutore a danno del minorenne tutelato.

In particolare nei reati commissivi, la qualifica di Tutore comporta la commissione di reati specifici, es. ex art. 609 quater c.p. la configurabilità del reato di atti sessuali con minorenne, altrimenti escluso dopo i 14 anni, nel più ampio limite dei 16 anni. 
Con riferimento ai reati omissivi: applicazione dell'art. 40 cp u.c. "Non impedire un evento, che si ha l'obbligo giuridico di impedire, equivale a cagionarlo" e l'art. 361 in materia di denuncia di reati perseguibili d'ufficio tra cui quello di cui può essere vittima o autore il minorenne, perché il tutore, sia quando pubblico che quando privato, secondo un orientamento estensivo della Corte di Cassazione Penale sent. 27570 del 12 luglio 2007, come in raccordo con la misura dell'Amministrazione di Sostegno Cass. Penale Sez. VI 12 novembre $2014 \mathrm{n}$. 50754 sarebbe soggetto obbligato in quanto incaricato di pubblico servizio.

Ed ancora tutti i reati in materia di rivelazione di segreto ed in materia di privacy, nonché la mancata esecuzione dolosa dell'ordine del giudice (art. 388 c.p.).

Si ricorda infine che il ruolo di tutore comporta l'applicazione ex art. 61 c.p. di un'aggravante generica, mentre ai sensi dell'art 609 ter (abuso sessuale su minore) un'aggravante specifica.

\section{I Diritti esigibili dal Minore Straniero non Accompagnato}

\section{a) Il Diritto al regolare soggiorno in Italia}

Tra i diritti a cui il minorenne straniero non accompagnato ha primaria necessità di accesso, oltre a quelli di natura sanitaria (per cui il Decreto del Presidente del Consiglio dei Ministri del 12 gennaio 2017 recante "Definizione e aggiornamento dei livelli essenziali di assistenza, di cui all'art. 1, comma 7, del Decreto legislativo 30 dicembre 1992, n. 502" che sancisce l'obbligo di iscrizione al SSN dei minori stranieri non accompagnati in condizioni di parità con i cittadini italiani) vi è quello in questione, che ricomprende le seguenti modalità per permanere regolarmente sul territorio italiano e pertanto nel Sistema di accoglienza e protezione collegato:

1) a seguito di richiesta di diritto a protezione internazionale (rifugio o protezione sussidiaria)

Procedura ed esito:

a) riconosce lo status di rifugiato (permesso di soggiorno di 5 anni rinnovabile) o la protezione sussidiaria (permesso di soggiorno di 5 anni rinnovabile)

b) rigetta la domanda qualora non sussistano i presupposti - viene ridimensionata la possibilità di rilascio di pds per motivi umanitari (a seguito della 1. 132/2018-conversione decreto sicurezza) o comunque si riceve un permesso per minore età.

2) fuori da una formale richiesta di asilo:

a) permesso per minore età o per tutela o affidamento 
In entrambi i casi il permesso potrà essere rinnovato alla maggiore età (previa sussistenza dei requisiti). In questo senso il decreto cd Salvini (n. 113/18) ha abrogato il permesso per motivi umanitari ed introdotto il $\mathrm{cd}$. permesso di soggiorno per motivi speciali.

Dal combinato disposto delle norme in materia di protezioni internazionale (rifugio o protezione sussidiaria) (D.lgs 140/2005, D.lgs 251/07, D.lgs 25/08 (mod. D.lgs 159/08), L. 47/17 (cd Legge Zampa), Decreto Minniti (1. 46/17 mod. D.lgs 142/2015), il minorenne straniero non accompagnato è comunque inserito nel percorso di accoglienza dei minorenni richiedenti asilo, anche se non dovesse formalizzare tale domanda .

Tale sistema è retto da progetti Ministeriali (struttura di missione) e Comunali (ex SPRAR- ora SIPROIMI) riservato solo ai minorenni.

\section{b) Altri Diritti consolidati ai MSNA}

Oltre ai diritti di permanenza di accesso alle cure sopra citati, esistono anche altri diritti di cui il minorenne straniero non accompagnato è titolare, alcuni dei quali sono anche "diritti chiave", perché consentono l'accesso, altrimenti impossibile o più complesso, ad altri diritti; ad esempio il diritto alla residenza, ad un'accoglienza a cui si collega un progetto formativo e/o scolastico, un'adeguata alfabetizzazione, ecc.

Tra questi diritti troviamo pertanto: inespellibilità, collocamento in luogo sicuro del minore, assistenza socio psicologica, sanitaria e orientamento legale, supporto di mediatori linguistico culturali, verifica della presenza di parenti, connazionali o persone disponibili ad una eventuale presa in carico del minore, apertura della tutela pubblica (Ente sociale del comune di accoglienza) presso il Giudice Tutelare in tempi certi, regolarizzazione dello status giuridico e della presenza sul territorio (anagrafe sanitaria, cittadina, residenza, ISEE, ecc.), iscrizione al Servizio Sanitario Nazionale, insegnamento di base della lingua italiana, inserimento scolastico e professionale e attivazione di servizi a sostegno dell'integrazione socio lavorativa del minore, definizione di un progetto socioeducativo individualizzato.

Altri diritti sono stati poi introdotti dalla L. 47/2017 e dal D.lgs. 220/17:

- La tutela privata/volontaria (con trasferimento competenze al TM e il nuovoruolodell'Autorità Garante perl'Infanzia el'Adolescenza);l'equiparazione trattamentale tra MSNA che presentano o meno l'istanza di protezione internazionale; procedura giudiziale definita per l'accertamento dell'età e rimpatrio assistito, misure di accoglienza diverse dalla comunità (es affidamento familiare), una maggiore attenzione al racconto migratorio e al progetto educativo rispetto alla provenienza, la quasi totalità dei richiedenti protezione internazionali MSNA. 


\section{I dati}

Analizzando una serie storica recente (2016-2020), costruita sui dati del Ministero del lavoro e delle Politiche Sociali ${ }^{6}$, e con un successivo focus sulla Regione Emilia Romagna, i minorenni presenti e censiti nella nostra regione sono passati da 1.081 del dicembre 2016 a 792 del 31 dicembre 2018 e, in termini percentuali, l'Emilia-Romagna rappresentava la quarta regione per accoglienza con quasi il 7,0\% rispetto al dato nazionale (10.787 al 31 dicembre 2018).

Al 29/2/2020 (medesima fonte), i MSNA presenti in Regione sono passati a 568 (un sostanziale dimezzamento in soli 3 anni), confermando l'Emilia Romagna ancora quale quarta regione per accoglienza (dopo Sicilia, Lombardia e Friuli Venezia Giulia), con il 10,6\% rispetto al dato nazionale (5.368).

- Parte di chi arriva si rende subito irreperibile e diventa oggetto di sfruttamento (maschile e femminile)

- Le forme di accoglienza comunitaria in qualche caso si trasformano anche in affidamento familiare

In Emilia Romagna, al 29/2/2020, provengono nel 30\% dei casi dall'Albania, nel 10\% dall'Egitto, nel 9, 3 \% dal Bangladesh, nel 7,5 \% dal Pakistan. Nell' $88 \%$ dei casi hanno almeno 16 anni.

Sono invece diminuiti drasticamente negli ultimi anni gli arrivi di cittadini dall'Africa sub-sahariana, tra cui Nigeria (per il ruolo strategico della Libia) e quelli che usavano la tratta balcanica (per il ruolo della Turchia, che accoglie ad oggi circa 4 milioni di rifugiati, di cui la gran parte dalla Siria ).

Il dato della nostra Regione è ancora più significativo se consideriamo la sola accoglienza femminile, dove è seconda (con il 14, $6 \%$ ) dopo la Sicilia (con il $23,8 \%)$.

Le nazionalità sono principalmente Nigeria $(21,2 \%)$, Albania $(18,1 \%)$ e Costa d'Avorio (11,9\%), per un dato nazionale di 260 presenze. Anche in questo caso, più del 66\% ha almeno 16 anni.

\section{Conclusioni}

La creazione di un Ufficio Tutele Metropolitano ha consentito di convogliare le istanze giuridiche dei Servizi Sociali dell'Area bolognese (c.ca 1 milione di abitanti) presso un unico "Ufficio comune".

Ciò ha peraltro consentito di unificare non solo la redazione delle consulenze legali ma anche dell' intero piano formativo rivolto agli operatori sociosanitari, con particolare riguardo alla materia minorile.

6 Fonte: https://www.lavoro.gov.it/temi-e-priorita/immigrazione/focus-on/minori-stranieri/Docume nts/Report-MSNA-mese-febbraio-2020.pdf 
Da ultimo ha reso più agevole i rapporti con le Autorità Giudiziarie competenti (Tribunale per i Minorenni, Giudice Tutelare, Tribunale Ordinario in sede collegiale).

La materia dei minori stranieri non accompagnati, che già aveva visto nel passato recente un modello metropolitano nell'erogazione delle prestazioni sociali, è stato anch'esso interessato da questo progetto giuridico in un'attività volta a bilanciare le azioni dovute ex lege con azioni volte a promuovere sempre più compiutamente i diritti esigibili. Di qui il ruolo sopra descritto del Tutore, pubblico o privato, e del suo stretto raccordo tanto con i Servizi Pubblici quanto con la società civile, le Autorità Giudiziarie e lo stesso beneficiario della forma di protezione giuridica.

La logica tutelare che indirizza questi sforzi, volendola vedere anche dal punto di vista contabile, parametro di cui deve comunque tenere conto una Pubblica Amministrazione, è quella di trasformare azioni che all'apparenza sembrerebbero voci di spesa/costi (in termini di risorse, di personale, organizzative, progettuali) in investimenti. Azioni che rafforzano il senso di sicurezza diffusa quali nuove forme di prevenzione, di coesione sociale, di partecipazione pubblica, del rafforzamento del tessuto connettivo cittadino e metropolitano attraverso un' integrazione partecipata. Un Welfare di comunità che sempre più può definirsi partecipativo ${ }^{8}$ e più recentemente inteso all' interno del paradigma generativo 9 .

Letture queste che dovranno orientare il lettore per meglio comprendere le azioni e le attività rivolte a sostenere, proteggere e tutelare gli infanti e gli adolescenti in difficoltà , tra cui i minorenni stranieri non accompagnati, fuori dai clichè ormai anacronistici dell'assistenzialismo, ritenendoli invece quali interventi (più o meno strutturati ed organici) rivolti a creare coesione sociale e a rigenerare le risorse già disponibili, sostenendo i soggetti più esposti a precarietà assistenziali e a situazioni di pregiudizio.

\section{Bibliography}

Ciceri G., Cau M., Maino G., Welfare dei servizi e welfare di comunità, Maggioli 2019. Gioncada M., Diritto dei servizi sociali. Per assistenti sociali, psicologi, educatori, avvocati, Maggioli 2012.

Lavedini G., Prime brevi considerazioni sul ruolo dell'Ente Locale nell' Amministrazione di Sostegno, "Il Diritto della Regione" 2005, No. 1-2.

7 G. Ciceri, M. Cau, G. Maino (a cura di), Welfare dei servizi e welfare di comunità, Maggioli 2019.

8 V. Pellegrino, Nuovi Spazi Istituenti. Il welfare partecipato come "bene comune", [in:] Animazione Sociale, Gruppo Abele 2014.

9 T. Vecchiato, Il Welfare generativo una sfida politica e sociale, "Studi Zancan" 2013, No. 2. p. 40, 2013. 
Pazè P., La Tutela e Curatela dei minori, [in:] Trattato di Diritto di Famiglia, ed. P. Zatti, Milano 2002.

Pellegrino V., Nuovi Spazi Istituenti. Il welfare partecipato come "bene comune", [in:] Animazione Sociale, Gruppo Abele 2014.

Report mensile minori stranieri non accompagnati (MSNA) in Italia. Dati al 29 febbraio 2020, Direzione Generale dell' Immigrazione e delle Polotiche di Integrazione Divisione II, https://www.lavoro.gov.it/temi-e-priorita/immigrazione/focuson/minori-stranieri/Documents/Report-MSNA-mese-febbraio-2020.pdf

Strumendo L., Intervento in materia tutelare, "Pubblicazione Difensore Civico Emilia Romagna Quaderno” 2011, No. 2.

Vecchiato T., Il Welfare generativo una sfida politica e sociale, "Studi Zancan" 2013, No. 2.

\section{SUMMARY}

Unaccompanied Foreign Minor, between consolidated rights and emerging protection needs. The point of view of an italian Metropolitan Protection Office

In the introduction, the legal system concerning reception and protection of unaccompanied foreign minors is analysed, especially, the Public Guardian. It is responsible for promoting and guaranteeing the rights of the protected child, not only of a conservative nature (preserving rights already acquired) but stimulating the protected (in classical civil language we would call it "pupil"). In the second paragraph, I analysed the importance of the public guardian as an entity separated from the person directly providing care and social assistance, in order to promote not only support but also a role of guarantee. Then the protection of unaccompanied foreign minors is the focus on my analysis. In particular, the Voluntary Guardian which represents a private mentor of an unaccompanied foreign minor. This figure takes care not only of the legal aspects but especially those aimed at the integration of the child in Italian society. In the 5th paragraph a legal examination on the institute of the protection is made with reference to the role of the guardian and the legal responsibilities. In addition to formal forms of protection, there are some levels of substantive legal protection that impose "challenges" for the local authority and also for the Guardian. These are therefore the "key" rights, because they allow access to other rights. They are fundamental "social" rights. Some public data on the flows of the last years are analysed with the aim to make conclusive remarks about the advantages deriving from the creation of the Metropolitan Public Protection Office of Bologna. 